\title{
EL USO DE MODELOS COMO INSTRUMENTOS PARA LA FORMULACIÓN DE POLÍTICAS DE POBLACIÓN
}

\author{
HaNnes Hyrenius \\ Universidad de Göteborg, Suecia*
}

\section{INTRODUCCIÓN}

EN los últimos AÑos, en las discusiones acerca del crecimiento de la población, el desarrollo y la expansión económica, la explotación de recursos naturales y los problemas del medio ambiente, los especialistas han comenzado a utilizar descripciones simplificadas de la realidad mediante el uso de modelos. El propósito de este trabajo es intentar hacer evidentes las implicaciones de estos problemas y, de ser posible, ampliar las oportunidades de la sociedad para la formulación de políticas relacionadas con aspectos económicos, sociales, demográficos, etc.

Puede considerarse que el desarrollo de modelos sobre las condiciones sociales y su evolución dio principio a partir de las elaboraciones de los métodos econométricos. Estos métodos centraron su atención y siguen concentrados principalmente en los fenómenos económicos propiamente tales. Los medios de ayuda fueron, al principio, de naturaleza matemática y estadística. Sin embargo, pudieron producirse modelos más generales, cuando las computadoras y las técnicas de manejo de datos comenzaron a emplearse como medios de asistencia.

Los primeros intentos de modelos del desarrollo social fueron determinísticos (no probabilísticos) en el sentido de que las relaciones y conexiones se consideraban determinadas. Esta restricción fue rápidamente superada cuando el trabajo se llevó a cabo a distintos niveles alternativos y con varios conjuntos de valores de parámetros. Sin embargo, las técnicas para el manejo de datos hicieron posible introducir variaciones en las componentes individuales por medio de distribuciones combinadas con técnicas estocásticas, así como también por adición de otros tipos de perturbaciones aleatorias.

Las distintas técnicas disponibles ahora han sido utilizadas en la construcción de modelos amplios con aspiraciones muy ambiciosas. Considerando a la sociedad humana como un sistema, los modelos incluyen, en

* Informe preparado para el Comité de Expertos Asesores de las Naciones Unidas sobre el Plan de Acción Mundial de Población. Traducción de Abelardo Hernández $M$. 
sus formas generales, los tres factores principales - recursos naturales, población y capital- así como un número creciente de fenómenos y subfactores.

La posibilidad de incluir en un modelo todas las condiciones y variables "esenciales" dependerá, por supuesto, del desarrollo posterior de la técnica empleada. Si la construcción de modelos sirve para propósitos prácticos de toma de decisiones y para la formulación de políticas debe juzgarse en cada fase la capacidad del instrumento, sus grados de generalidad, su sensibilidad y su incertidumbre (márgenes de error). Asimismo, debe juzgarse qué tan útil es el método en el estudio de los diferentes sectores y áreas de la sociedad global.

El desarrollo de modelos presupone una colaboración estrecha entre disciplinas diferentes tanto en su naturaleza metodológica como en su naturaleza sustancial. Una acción recíproca ha tomado lugar aquí y aún esfuerzos parciales, insuficientes y defectuosos han tenido un efecto estimulante en el desarrollo de los métodos. Al mismo tiempo, se ha producido un interés creciente en los círculos políticamente responsables y en el público en general.

El propósito del presente estudio es poner de manifiesto si los tipos de modelos desarrollados hasta ahora $\longrightarrow$ tipos que pueden ser concebidos actualmente - pueden ser utilizados como instrumentos en la formulación de políticas de población.

De aquí surgen dos preguntas:

Primera: ¿cómo se define una política de población?, ¿cuáles son los factores demográficos y los procesos sobre los que queremos influir y qué fenómenos y procesos vinculados a la población deberían incluirse bajo el nombre de política económica, política social, etc.? Segunda: ¿qué propiedades buscamos en un instrumento si nuestro propósito es elucidar y comparar los efectos que diferentes alternativas tienen sobre nuestra política de población?

Se ha hecho ya un examen más detallado de lo que puede razonablemente ser demandado de un modelo de acuerdo con diferentes definiciones de lo que se considera corresponde a una política de población. Como se esperaba, el estudio mostró que los factores demográficos están tan íntimamente relacionados con las condiciones económicas, sociales y otras, que aun la mínima demarcación de una política de población requiere que las relaciones mencionadas sean tomadas en cuenta debidamente. Prescindiendo de cómo se desea formular la noción de política de población, esto conduce, a su vez, a una demanda de lo que describiremos en la sección siguiente en relación con las definiciones de modelos conocidos como "modelos dinámicos demográfico-económico-sociales comprehensivos" (MODDES).

Por lo que se refiere a las propiedades requeridas por los MoDDes, sólo podemos hacer algunas observaciones aquí. Ulteriores puntos de vista serán presentados en las secciones siguientes.

$\mathrm{El}$ interjuego entre los distintos factores debe mirarse como un sistema del cual se sigue: 
1. que deben incluirse todos los factores que se juzguen necesarios de acuerdo a criterios determinados;

2. que dichos factores se encuentran indicados y medidos de acuerdo a mediciones correctas (insesgadas);

3. que las relaciones y los circuitos de retroalimentación sean incluidos correctamente y en la medida necesaria, y;

4. que todas las constantes, parámetros, relaciones y retroalimentaciones sean cuantificadas en forma satisfactoria.

Por supuesto, lo que cada uno de estos requerimientos significa en particular no será explicado aquí. Sin embargo, puede anotarse que el grado de alcance puede variar de acuerdo con los problemas que sean de interés para usos y aplicaciones separadas del modelo.

\section{DEFINiCiones DE MODELOS}

La palabra "modelo" se ha usado, progresivamente, con poca variación en su significado. Desde siempre, el término fue usado para designar únicamente los modelos concretos que los constructores de edificios, puentes, etc., utilizaban para realizarlos de manera adecuada. Se trataba entonces, casi, de un asunto en el que las miniaturas a escala ayudaban a concebir la versión final de tamaño real. Con el mismo término se denominaba también a las miniaturas y diseños usados por cierta clase de artistas. El polo opuesto a esta concepción de la palabra "modelo", se encuentra en la costumbre de igualar su significado con las teorías puras, expresadas en forma verbal. Sin embargo, tal significado es más comprensible cuando las teorías se establecen mediante símbolos y expresiones más precisas. Aquí entra en escena el lenguaje matemático, pero la dislinción entre un "modelo" y un proceso de análisis deductivo lógicomatemático, resulta a veces poco clara.

Cuando la utilización de la palabra "modelo" se impuso en el análisis propio de las ciencias sociales, se apoyaba principalmente en conceptos y relaciones del campo económico que podían ser formulados cuantitativamente. De aquí que la econometría se haya desarrollado como una rama independiente de esta ciencia. No fue sino hasta un poco más tarde que tuvo lugar un desarrollo similar en el tratamiento de otros aspectos de la vida social (pautas de comportamiento social, factores demográficos, planeación social, fenómenos de geografía cultural, etc.) .

Puede decirse que el propósito principal de la construcción de un modelo es reunir, en forma simplificada, los componentes más importantes (factores y variables) de los fenómenos más complejos que se pretende estudiar. Así, se pueden evidenciar las cualidades y las relaciones esenciales y generales ("estructura", "esqueleto"). Aquí, es necesario enfrentar, en principio, varias tareas:

1) definición y delimitación del campo de estudio;

2) determinación de los componentes (factores) que serán incluidos en el modelo (la construcción); 
3) Técnicas de medición y niveles de especificación;

4) relaciones lógicas y reales entre los factores, incluyendo las "relaciones de retroalimentación";

5) clasificación de factores no numéricos. Clasificación y cuantificación de variables numéricas; y

6) métodos de prueba de la precisión (confiabilidad), sensibilidad y estabilidad del modelo, mediante material histórico y elaborado.

De acuerdo a su naturaleza, un modelo puede ser estático o no estático. A la primera categoría pertenecen, naturalmente, todos los modelos concretos (miniaturas), así como también las teorías que pueden ser expresadas por leyes o que pueden indicar con exactitud determinada la forma en que un fenómeno depende de otro. En principio, esta clase de modelos puede ser intercambiable con, o expresable por medio de conjuntos de constantes.

Puede decirse que los modelos no estáticos dan una expresión simplificada de los procesos que se continúan en el tiempo con más o menos claridad. Esto puede considerarse también como un fenómeno complejo que actúa en una dimensión temporal, y en consecuencia puede ser tratado, en parte, como un conjunto acoplado de series de tiempo.

De acuerdo con la terminología usada en teoría econométrica y demométrica, se puede hablar paralelamente de modelos estacionarios, estables, y dinámicos. Los modelos estacionarios deben ser interpretados en términos de la permanencia, en conjunto, de las condiciones en el largo plazo, aunque puedan ocurrir variaciones de plazo corto, en parte como oscilaciones aieatorias observables y en parte como variaciones cíclicas de diferente longitud de onda y potencia respecto de un nivel de referencia. Se dice que un modelo es estable si sus factores de cambio son, en forma similar, mantenidos constantes (estacionarios) sin que su resultado neto implique equilibrio.

Un ejemplo de esto último puede encontrarse en el proceso reproductivo del hombre. Se ha demostrado que la existencia de una discrepancia entre fecundidad y mortalidad en el tiempo, conduce a una estructura definida de la población, pero bajo el supuesto de crecimiento constante (" $p$-estructura estable"). Si en casos excepcionales llega a producirse el equilibrio, se obtendrán las condiciones estacionarias ("o-estabilidad"). Por supuesto, ésta es sólo una especulación teórica, dado que las variables de cambio, mortalidad y fecundidad, nunca permanecen constantes durante un período de tiempo suficientemente largo (excepto, parcialmente, bajo condiciones muy primitivas). Además, estos hallazgos no pueden ser aplicados automáticamente a otros sectores de la sociedad, aunque puedan existir algunos paralelismos.

La expresión dinámico puede usarse para modelos siempre que éstos estén basados en cambios en el tiempo, independientemente de que tales cambios puedan expresarse como variaciones regulares, o de que estén vinculados tho a otro como consecuencia de retrasos necesarios en el 
tiempo entre los estímulos y las respuestas. Debe notarse que la expresión "dinámico" es entonces un término muy general que, en principio, incluye a todos los modelos no estáticos.

Debería observarse, además, que algunos fenómenos que actúan en un período limitado, aparecen formalmente como series de tiempo, aunque el tiempo no sea de interés. Tales cambios/procesos pueden a veces referirse mejor a su dependencia que a la duración del fenómeno, en la medida en que pueden repetirse, al margen de su ubicación precisa sobre un eje de tiempo seleccionado.

Dentro del esquema del campo de estudio establecido, un modelo puede ser general o parcial (o sectorial). La delimitación será por supuesto clara o indistinta, dependiendo de la posibilidad de deslindar, con más o menos certeza, lo que pertenece al campo de estudio en cuestión.

Un modelo general debería incluir, en principio, todos los factores variables esenciales, así como las cualidades que de él se juzgue necesario incluir en el "sistema". Lo que es excluido, consciente o inconscientemente, puede considerarse entonces como una perturbación externa. Si éstas tienen un efecto más extensivo pero no obstante son dejadas de lado, pueden ser descritas como "modificadores" o "multiplicadores" cuyo efecto es mantenido confinado o fijo en uno o más niveles.

Cuando se desea estudiar con detalle cierto fenómeno dentro del campo de estudio, para poder hablar de un modelo sectorial o parcial, es necesario que esto se haga bajo ciertos supuestos simplificados. En efecto, debe hacerse una delimitación nueva y más rigurosa del campo de estudio o, en otras palabras, crear un subcampo. Esto constituye una analogía respecto al caso antes mencionado: las relaciones entre el campo parcialsectorial y el fenómeno no incluido en el sistema más amplio son entonces considerados como perturbaciones o modificadores, aunque de naturaleza más cercana.

Como un ejemplo de lo que se está tratando de decir, supongamos que el campo demográfico ha sido escogido como campo general de estudio, y dentro de él, algunas variables convencionales (mortalidad, nupcialidad, fecundidad, migración, etc.). Pueden considerarse, como modificadores diferentes factores y condiciones sociales, económicas y culturales, etc. Si se desea entonces limitarse a un campo específico de estudio, digamos la fecundidad, es claro que la nupcialidad, la separación y la estructura demográfica, etc., entrarán en escena con la categoría inmediata de modificadores.

Partiendo de la definición del campo de estudio, el alcance que ésta debe cubrir, puede, por supuesto, variar en magnitud. La palabra "general" anterior, no intentaba referirse a alguna comprensión acabada del tema. Aquí, por lo tanto, caracterizaremos como modelos comprehensivos a los casos en los que haya un intento por incluir dentro del campo de estudio todos aquellos factores y relaciones cuya naturaleza y magnitud son tales que su acción tiene un efecto significativo sobre las cantidades y ocurrencias derivadas del análisis (y su posible predicción).

Finalmente, debería mencionarse que el término "modelo especial" 
es usado a veces para describir modelos parciales que están sujetos a ciertas condiciones o restricciones, o también a alguna expansión especial. Podemos tomar como ejemplo un modelo sectorial de la reproducción de la población humana. Éste necesita ser modificado para incluir la fecundidad de, por ejemplo, algún grupo de población. Lo mismo tendría que hacerse si va a ser usado en circunstancias de vida humana sujeta a condiciones climáticas extremas.

Puede hablarse de un modelo deterministico o estocástico, aunque tal término se aplica con más propiedad a las componentes del modelo y a las formas de operación que son caracterizadas por estas expresiones. En el primer caso, se trabaja con valores constantes de las variables incluidas y con las relaciones fijadas entre ellas. En el segundo caso, un factor se deja variar de acuerdo a ciertas reglas, en general por medio de una distribución combinada con un proceso aleatorio.

Una simple transición de la forma puramente determinística es obtenida ubicando en el nivel de una variable y de sus relaciones con otras variables, un número de valores alternativos en proporciones prescritas. En general, un modelo utiliza tanto elementos fijos como estocásticos, y puede, en consecuencia, ser descrito como mixto. Por supuesto, la fijación de los valores es particularmente adecuada cuando el fenómeno tiene poca variación o cuando, además, la variación tiene poco efecto. Como un ejemplo simple puede mencionarse la tasa de fecundidad por sexo.

El término simulación tiene varios significados. El significado original se refiere al hecho de imitar una condición o un curso de eventos. En el trabajo con modelos, esto significa que se hace una "corrida" con todas las variables controladas y distribuidas en "valores de entrada" específicos. En un modelo determinístico, el resultado final viene entonces a ser el mismo en cada corrida. Con la presencia de elementos estocásticos, o de simples perturbaciones aleatorias, se obtendrán resultados diferentes para cada corrida.

En modelos expresados matemáticamente o en algunos de sus aspectos, el proceso estocástico es incluido directamente en la construcción del modelo. Cuando se formula un programa de computación, la aleatorización es llevada a cabo por medio de una técnica basada en números aleatorios producidos por una computadora o algo equivalente a esto.

Cuando un modelo se basa en agregados (población total, fuerza de trabajo total, acervo de capital, oferta de energía, recursos naturales, nivel general o medio de consumo de alimentos, etc.), el modelo es llamado macromodelo. Si se basa en unidades separadas (empresas, familias, personas individuales, máquinas separadas, etc.) el modelo será llamado micromodelo.

Cuando un macromodelo es puesto en operación, su flexibilidad dependerá, en medida considerable, de la manera en que sean incorporados los ingredientes estocásticos. El campo de variación abarca desde una estructura muy compacta con apariencia casi estática o estacionaria en tanto que su funcionamiento permita una amplia variación en las diferentes componentes y sus interrelaciones. Cuando las operaciones aleato- 
rias son aplicadas a unidades individuales, digamos los individuos humanos, hablamos de micromodelos de simulación.

Si en un modelo de microsimulación, un número suficiente de corridas se hace conjuntamente, los valores medios de las diferentes variables resultantes serán similares a los resultantes de un macromodelo. Sin embargo, los resultados contendrán también razgos que no podrían obtenerse en una macro-aproximación directa, en especial información acerca de la variación en las variables resultantes y sobre la variación en sus relaciones. Esto último es importante cuando se trata de medir la sensibilidad del modelo y cuando se estudian intervalos de confianza en las predicciones.

En las aplicaciones de modelos, el aspecto espacial lleva a una división entre modelos globales, regionales, nacionales y locales. Parece deseable, en relación con esto, evitar la palabra "total" en las aplicaciones globales, dado que ésta también podría referirse en esencia a la cobertura denominada antes como "general".

En cierto sentido, las demandas de un modelo global son menores que las de un modelo regional desde el momento en que en el primero no se toman en cuenta los flujos externos de materias primas, bienes, fuerza de trabajo, población inactiva, capital, conocimiento técnico, etc. Por otro lado, si se espera que un modelo global tome en cuenta la heterogeneidad de la sociedad y la población, debe existir en él un cierto grado de diferenciación y de detalle, el cual algunas veces puede evitarse, al menos parcialmente, en modelos formulados para aplicaciones regionales y locales, mientras mantengan el mismo nivel de aspiración.

Sin embargo, los problemas son más complicados. Aún un modelo global tiene que incluir ciertas expresiones en las que se considere la heterogeneidad -por ejemplo, rural-urbana y cambios internos y externos de la fuerza de trabajo- y esto, a su vez, presupone métodos de tratamiento de trasposición de una categoría a otra.

Por último, puede enfatizarse que un modelo debe siempre, por supuesto, hacer un compromiso entre una reproducción detallada y realista de la realidad, por un lado, y la necesidad de manipulación de la misma, por el otro. La micro-simulación de la población, el capital y los recursos naturales, no es factible de llevarse a cabo simultáneamente. Sin embargo, puede recurrirse a la desagregación que, de acuerdo con criterios apropiados, descompone la heterogeneidad del mundo que se está reproduciendo, en lo que llamaremos aquí submodelos de condiciones homogéneas. La desagregación debe tener varios propósitos:

a) clasificación geográfica;

b) división de acuerdo con las condiciones demográficas;

c) separación en dos o más estratos de acuerdo con los grados de desarrollo de la sociedad.

Una combinación de éstos, y de otros aspectos, plantea dificultades de varios tipos. En primer lugar, se necesitan criterios para llevar a cabo una división óptima en estratos homogéneos. Aquí, los datos estadísticos aparecerán como altamente insatisfactorios, dado que en general no lle- 
gan a cubrir el campo y los estratos que uno desea distinguir. En segundo lugar, se requieren métodos avanzados para tratar con flujos de personas, recursos y capitales. En tercer lugar, surge la necesidad de hacer cuantificaciones realistas dentro del modelo, para todos los estratos homogéneos que han sido definidos.

\section{REVISIÓN DE MODELOS SELECCIONADOS}

\section{Discusión general}

En los últimos tiempos se ha desarrollado un gran arsenal de métodos y de técnicas científicas para su uso en el análisis de aspectos económicos, sociales, demográficos y otros, de la sociedad humana. Los métodos estadísticos, desde luego, han jugado un papel importante en la descripción y explicación de las condiciones y tendencias, pasadas y presentes. Se han analizado datos agregados $\mathrm{y}$, parcialmente han sido ampliados mediante extrapolaciones para predicciones de distintos tipos.

Se han recogido datos a niveles micro (individuos, familias, viviendas, empresas), a través de procedimientos de muestreo cada vez más avanzados, con el propósito de ampliar nuestro conocimiento sobre las tendencias y las situaciones actuales $\mathrm{y}$, como fin último, para establecer bases para la acción política en asuntos económicos, sociales, educacionales, de salud, culturales, etc.

Todos estos métodos y técnicas, por supuesto, serán utilizados en el futuro, y se desarrollarán aún más para cumplir con nuevos propósitos. A este respecto, las técnicas de computación incrementarán su capacidad para procesar tales análisis. Además, todo ello of rece nuevas posibilidades de conocimiento coordinado de muchas áreas, conjuntamente con el manejo de grandes masas de datos numéricos. Esto es importante debido a que los métodos "convencionales" se ven limitados a unos cuantos factores en una sola vez.

Como ejemplo, pueden mencionarse las investigaciones acerca de las condiciones de salud, en donde los estudios se han limitado a la estratificación con base en uno o dos factores a la vez, entre ingreso, profesión, grupo social, nivel de educación, etc. Usando una computadora, no sólo se pueden agregar otras formas de estratificación, y manejar varias de ellas simultáneamente, sino que también puede estudiarse el interjuego de los distintos factores con mayor sentido que antes. Con los procedimientos estadísticos convencionales, las fuentes de variación pueden ser separadas -y eliminadas - en la variable bajo investigación. Sin embargo, las técnicas de computación modernas hacen posible también distinguir y medir los efectos "secundarios" de tal factor.

Un enfoque realista para analizar el complejo mundo de la realidad, lleva, como ya se ha indicado en la introducción, a la disyuntiva de decidir qué factores deben incluirse y a la definición de un sistema de variables, con sus condiciones y relaciones. Las interrelaciones entre factores son consideradas con más ciudado por los llamados circuitos de 
retroalimentación. Esstos deben basarse en un profundo conocimiento del fenómeno y deben formularse, cuantificarse y probarse en términos de valores observados, y posiblemente, donde los datos sean deficientes, con valores supuestos.

Estas consideraciones describen brevemente el enfoque moderno en distintas áreas del conocimiento. Para los propósitos específicos de las políticas socio-económicas y, particularmente, en acciones encaminadas hacia asuntos poblacionales y problemas relacionados con la población, se han desarrollado técnicas tanto para propósitos especiales (limitados) como para propósitos más generales.

Nuestro trabajo presente será restringido en ciertos aspectos. De esta manera no discutiremos el papel de los métodos estadísticos, aunque éstos cubran en el presente un amplio rango de técnicas especialmente desarrolladas para satisfacer las necesidades de análisis en los problemas socio-económicos. Respecto a los "modelos", no entraremos aquí en la presentación o crítica de otros campos específicos que no sean el de la población (considerada en sentido amplio).

Como se apuntó a manera de introducción, los distintos factores de la población están, en general, estrechamente relacionados con factores económicos, sociales, culturales, religiosos, etc., de modo que no pueden menospreciarse al formularse medidas sociales tendientes a influir sobre los mismos factores de la población. Agréguese a esto el hecho de que por medio de tales medidas, una vez que han sido puestas en operación, se producen, en grado considerable, repercusiones sobre los otros aspectos de la vida social, directamente o, después de un cierto lapso, indirectamente.

Estas circunstancias llevan al requisito de que si va a ser posible desarrollar la estructura del modelo con instrumentos analíticos de formulación de políticas, éstos deben ser del tipo sofisticado multifactorial, que hemos descrito en la sección II como modelos demográfico-económicosociales, MODDEs, extensivos y dinámicos.

\section{Los modelos del IтM}

Si hacemos caso omiso de los esfuerzos realizados en relación con la investigación econométrica para desarrollar modelos más generales -que podrían ser descritos mejor como parciales- el primer intento de largo alcance parece ser el que posteriormente fue llamado el modelo de "los límites del crecimiento".

La iniciativa fue tomada por un grupo formado en 1968, "E1 Club de Roma". Es ésta una organización informal cuyo objetivo es promover el interés y el conocimiento de los factores fundamentales de nuestro sistema de vida, así como sus interacciones simultáneas -factores de naturaleza económica, social, política, física y biológica- y centrar la atención de políticos y público en general sobre ellos. En la búsqueda de formas adecuadas para esclarecer el actual y futuro "predicamento de la humanidad", se ha decidido centrar la atención en el método de "Análisis 
de sistemas", desarrollado por el Instituto de Tecnología de Massachusetts (ITM). Estos métodos, que desarrolló en primer término el profesor Jay Forrester, fueron originalmente diseñados para resolver problemas de transacciones económicas, pero pueden aplicarse también a otros campos.

El método consiste simplemente en que las relaciones que se consideran válidas entre las diferentes variables y factores incluidos en "el sistema", son programados para computador. Una técnica importante consiste aquí en el uso de "circuitos de retroalimentación", por medio de los cuales las interrelaciones son dadas en forma de un cierto número de "modificadores". Si las distintas relaciones son distribuciones observadas o valores cuantitativos postulados, el sistema puede conducir a la reproducción de un mundo más o menos "realístico".

El primer bosquejo que hizo Forrester de un Modelo Mundial comprendía un número limitado de factores básicos (población, recursos naturales, producción de alimentos, producción industrial, capital, contaminación y sobrepoblación). Después de algunas modificaciones y ampliaciones, un modelo "Mundo 2" fue publicado por este mismo autor en su libro Dinámica mundial.

Bajo la dirección del profesor Dennis Meadows, un equipo continuó el trabajo y construyó los distintos subsectores en sus diferentes aspectos. También se intensificaron los esfuerzos para cuantificar las condiciones y relaciones involucradas. Los resultados fueron reportados en la primavera de 1972 en un libro "popular" con el título de Los límites del crecimiento. Su publicación produjo una enorme y vasta discusión. El problema vino a ser de interés general. Parte de la crítica errónea que apareció se debió probablemente al hecho de que el reporte técnico no se incluía. Aún después de un año y medio se dispone solamente de un número restringido de copias de este reporte, en forma mimeografiada.

La crítica contra los modelos del ITM proviene de diferentes ramas de la ciencia. Una relación amplia de ellas ha sido presentada por un equipo de investigación de la Universidad de Sussex, en el libro Una critica a los limites del crecimiento (versión norteamericana, Models of Doom), el cual damos como referencia al lector. En conexión con esto, a continuación haremos algunas observaciones breves.

Las críticas emitidas por diversas personas e instituciones incluyen un gran número de sugerencias constructivas acerca de cómo mejorar los modelos, conservando la técnica del análisis de sistemas, o utilizando otros métodos. Como resultado se han iniciado un número de nuevos estudios e investigaciones.

En ésta y en una sección subsecuente comentaremos brevemente el modelo de Los límites del crecimiento y su sector de población. Una sección siguiente dará breve cuenta de un número de proyectos abordados como una consecuencia parcial del modelo. Nuestra selección ha dependido, en parte, del material disponible durante la elaboración de este informe.

Los propios autores de Los límites del crecimiento apuntan que el comportamiento del modelo debe estar razonablemente de acuerdo con 
el comportamiento del mundo real. Esto implica la necesidad de reunir datos cronológicos acerca de las variables del mundo real desde 1900 hasta 1970. El examen realizado por el equipo de Sussex "indica que los modelos del ITM no satisfacen del todo este requerimiento. Éste es parcialmente un problema de información y parcialmente una cuestión de relación entre fenómenos".

En el estudio de Sussex cada sector ha sido examinado por especialistas y se expone en seis capítulos. En un ensayo introductorio, "Malthus con una computadora", hecho por Christopher Freeman, se afirma (p. 7 en la versión norteamericana): "Con la excepción del sector de la población, éstos (los supuestos) son generalmente insatisfactorios". Este enunciado, por supuesto, es hecho con diferentes niveles de alcance para los diferentes sectores.

El juicio derivado del estudio de Sussex de que el sector población es satisfactorio es, en mi opinión, poco aceptable, como mostraremos en la sección siguiente. Deben establecerse requerimientos especiales en particular para la población y, por lo tanto, el sector población también debe considerarse insatisfactorio, aunque éste sea mejor y esté más detallado que lo que aparece en el texto del informe que constituye Los límites del crecimiento.

Los modelos del IтM se caracterizan por lo siguiente (Cole en el informe Sussex) :

1. el mundo es considerado como un sistema sin influencias externas;

2. selección de los principales factores y sus interrelaciones;

3. uso de valores medios agregados y cuantificación de relaciones hechas subjetivamente; $\mathrm{y}$

4. naturaleza no probabilística del modelo.

E1 análisis del grupo de Sussex es conducido por caminos alternativos, en función de los cuales se hace patente que el método de análisis de sistemas es altamente sensible a los cambios de los valores de los insumos. De esto se sigue también que la generalización burda, las deficiencias y los errores directos en la construcción de las relaciones y en la cuantificación pueden ser grandes y, posiblemente, de consecuencias completamente imprevistas.

Para el "Mundo 3", el informe Sussex expone los resultados con una división de las partes desarrolladas y de las partes en desarrollo. Se señala también que las diferencias que aparecen a lo largo de estos ejercicios no necesariamente llevan al rechazo automático de todos los subsistemas. Dado que la sensibilidad del análisis de sistemas es muy grande, una ampliación más cuidadosa de las interrelaciones y realimentaciones debería ser capaz de corregir ciertas deficiencias.

Un gran número de propósitos constructivos son adelantados en los distintos capítulos, y permiten su perfeccionamiento y, en parte, la división de proyectos.

Los siguientes puntos pueden considerarse como un juicio comprehensivo, y constituir objetos de contribución a la investigación: 
1. La agregación es bastante fuerte y puede empañar o aun hacer desaparecer la heterogeneidad en todos los sectores. Al mismo tiempo, vuelve difícil o imposible una ponderación de los distintos factores de cambio que siguen a una extrapolación aceptable.

2. En la estructura del modelo se encuentra ausente un cierto número de factores básicos esenciales, tales como estructura social, nivel técnico, nivel educativo, morbilidad y, no menos importante, un sistema de valores y un mecanismo de precios que puedan cubrir los hechos esenciales del proceso económico y del comportamiento social.

3. Por su diseño el modelo es dinámico, pero en algunos aspectos es realmente estático o casi-estático. Ésta es precisamente la implicación del hecho de que ciertos componentes esenciales de la sociedad humana no estén presentes.

4. El crecimiento geométrico exponencial asignado a uno o más componentes conduce necesariamente a situaciones catastróficas, aun si la tasa de crecimiento es en sí misma pequeña. Esto se aplica tanto al crecimiento de la población como al crecimiento de la producción industrial; se aplica a bienes individuales y se aplica al crecimiento de especies de animales y plantas.

5. Las técnicas usadas en la medición de los distintos fenómenos son pobres y poco confiables en algunas partes del modelo.

6. Las pruebas y los ajustes hechos se realizan con base en unidades demasiado concentradas y muy heterogéneas; carecen por lo tanto, en grado inapreciable, de significado para juzgar la validez y la capacidad de los modelos.

7. Deben ser dedicados esfuerzos especiales al desarrollo de métodos que incluyan diferentes clases de flujos de ingredientes, bienes, fuerza de trabajo, población inactiva, capital, etc.

8. Deben insertarse modelos especiales de micro-tipos, por etapas, en todos los sectores posibles con miras a ampliar la desagregación y precisión de la estructura interna.

\section{El sector población en el modelo "Mundo 3"}

Dado que la población es el centro del trabajo del modelo, es de particular importancia para el sector población, que éste sea bien construido y cuantificado. De hecho, esto es de importancia primordial para el modelo total por razones de organización del sistema, y no menos como consecuencia de la gran sensibilidad del análisis de sistemas ante errores en la construcción misma, deficiencias y generalizaciones en la medición del fenómeno, errores en la cuantificación y omisiones de subfactores y retroalimentaciones. El sector población ha sido criticado entre otros por el grupo de Sussex y por el Instituto de Investigación Demográfica de Ghotenburg. Nos limitaremos aquí solamente a hacer algunas observaciones.

Los primeros modelos ITM no hacían uso, del todo, de algún grupo de edades. En consecuencia, los nacimientos y las defunciones estaban 
expresadas solamente en tasas brutas. Además, la fuerza de trabajo no podía ser medida en forma adecuada. En modelos subsecuentes ("Mundo 3", base de Los limites del crecimiento) se han hecho varias mejoras. Sin embargo, la manipulación de factores demográficos deja todavía mucho que desear, si se juzgan con base en las aspiraciones moderadas de las técnicas demográficas.

La mortalidad es expresada solamente por un parámetro, la esperanza de vida (esperanza media de vida al nacimiento). De una "esperanza de vida normal" de 28 años, se suponen cuatro multiplicadores para tomar en cuenta la influencia de las condiciones de vida (alimentación, servicios de salud, hacinamiento). Esta influencia es verificada en una forma complicada y controversial.

La fecundidad es tratada a través de una interesante combinación entre la proporción de mujeres fértiles y la fecundidad total, esta última construida con base en la fecundidad total deseada y la efectividad del control de nacimientos. Los multiplicadores de las condiciones de vida están basados en la influencia de la producción industrial y en la esperanza de vida observada. Aun si la estructura lógica de esta aproximación fuera aceptable como principio, es difícil entender cómo una cuantificación de las distintas relaciones puede ser algo mejor que una serie subjetiva de informaciones reunidas de acuerdo a condiciones heterogéneas. Una combinación debe, por lo tanto, presentar riesgos de errores de diversas clases.

Para una discusión más detallada puede hacerse referencia al informe de Sussex. Resumiremos la capacidad del modelo mediante una simple comparación del desarrollo de la población 1900-1970 de acuerdo con estimaciones oficiales de Naciones Unidas y aquellas derivadas del "Mundo 3' (corrida stándard; véase el cuadro 1).

Cuadro 1

Población mundial, 1900-1970

\begin{tabular}{|c|c|c|c|c|}
\hline \multirow{2}{*}{ Año } & \multicolumn{2}{|c|}{$\begin{array}{l}\text { Poblacion mundjial } \\
\text { estimada }\end{array}$} & \multicolumn{2}{|c|}{ Incremento anual $(\%)$} \\
\hline & Naoiones Unidas & $\begin{array}{l}\text { Modelo } \\
\text { Mundo } 3 "\end{array}$ & Naciones Unidas & $\begin{array}{l}\text { Modelo } \\
\text { "Mundo } 3 "\end{array}$ \\
\hline 1900 & 1650 & 1610 & & 080 \\
\hline 1910 & - & 1760 & 0.50 & $\begin{array}{l}0.09 \\
0.82\end{array}$ \\
\hline $\begin{array}{l}1920 \\
1930\end{array}$ & $\begin{array}{l}1860 \\
2069\end{array}$ & $\begin{array}{l}1910 \\
2080\end{array}$ & 1.06 & 0.85 \\
\hline 1940 & 2295 & 2380 & 1.04 & 1.35 \\
\hline 1950 & 2486 & 2740 & $\begin{array}{l}0.80 \\
1.85\end{array}$ & $\begin{array}{l}1.41 \\
1.58\end{array}$ \\
\hline $\begin{array}{l}1960 \\
1970\end{array}$ & $\begin{array}{l}2991 \\
3632\end{array}$ & $\begin{array}{l}3210 \\
3790\end{array}$ & 1.94 & 1.66 \\
\hline
\end{tabular}

a Millones de personas. 
Debe recordarse que el modelo fue manejado para que se adecuara a algunos datos iniciales. A pesar de esto, puede decirse que el arreglo fue un tanto pobre. Utilizar el modelo para pronosticar, después de 1970, sería por tanto una aventura arriesgada.

De acuerdo con el modelo, las tasas de nacimiento y de defunciones que han dado como resultado este crecimiento de la población se comportan en forma muy peculiar, y se desvían en medida considerable de lo conocido (o de lo que se cree conocer para el comienzo del período).

El modelo "Mundo 3" toma en cuenta la edad en tres formas diferentes. O no se hace división alguna por edades, o bien se distinguen cuatro grandes grupos de edades, o 15 grupos de edades. Se consigue, naturalmente, un cierto mejoramiento con la diferenciación de la edad donde las mediciones de la natalidad y la mortalidad son pertinentes; sin embargo, el hecho de que el resultado no pueda considerarse como satisfactorio, se debe atribuir a la construcción y técnicas de medición, a la organización del multiplicador y a su cuantificación. Efectos dignos de señalar, y a veces extraños, se obtienen mediante el uso de una técnica especial de discontinuidad.

En el modelo, la fuerza de trabajo se calcula en 0.75 de la población total entre 15 y 65 años. Realmente, la fuerza de trabajo no es utilizada como un factor integrado en una función producción, sino que se incluye sólo en forma secundaria en un "sector de empleo" dentro del subsistema capital. Los autores explican que a nivel agregado hay siempre una fuerza de trabajo excedente por lo que entonces las fuerzas de trabajo resultan de poca significación en comparación con la generación de capital. Esta observación poco realista es acompañada por la consideración de que existen posibilidades ilimitadas de migración en el mundo. La calidad del trabajo no se toma en cuenta.

Esta forma de tratar la producción, que por supuesto, resulta de vital importancia para el sistema global ha sido criticada fuertemente. Nosotros remitimos al lector a una parte del informe de Sussex (P. A. Julien, Chr. Freeman, C. M. Cooper) .

Con tal formulación del sistema económico, por supuesto, existe poca capacidad para medir la fuerza de trabajo correctamente. Naturalmente, una aproximación más realista debe tomar en cuenta la restringida movilidad (inmovilidad parcial) de la fuerza de trabajo (y con ello, de hecho, la de la población total), junto con su "calidad" psíquica y física. Esto presupone una distribución de la población por sexo y por edad para las mujeres, además de su estado marital y tipo de familia-hogar, condiciones de salud (delimitación de la morbilidad e invalidez), escuela y educación ocupacional, etc. Todo esto está ausente en "Mundo 3", pero parece posible que estos aspectos podrían construirse sucesivamente en un diseño de modelo más diferenciado de sociedades/poblaciones homogéneas.

En su comentario sobre el modelo "Mundo 2", el grupo de Sussex finaliza la discusión de los subsistemas de población señalando:

"En realidad, el modelo no pronostica el tamaño más probable de la población mundial. Más bien el subsistema de población es un medio para 
generar cifras plausibles de población para usarlas como insumos de otros subsistemas - y, como tales, podrían haber sido hechas de manera más simple."

En opinión del autor, esto sería como colocar el carro adelante del caballo. El factor humano debe ser el alfa y omega del modelo en el sistema.

Desde un punto de vista demográfico, una distribución detallada por edades (grupos quinquenales) se hace necesaria, por supuesto, y debería ser ampliada hasta edades avanzadas. Para el estudio del empleo, tendría que hacerse una diferenciación por sexo; además, la estructura familiahogar debería manejarse en una forma adecuada, de manera que la participación femenina en la actividad productiva pueda tomarse en cuenta dentro del modelo.

Para calcular el consumo de bienes y servicios debe hacerse también una diferenciación de la población por edades, dado que la mayoría de los elementos se especifican aquí por edades y, en parte, también, por sexo y familia. Por lo que toca a los servicios, podemos anotar, por ejemplo, el cuidado de los niños y de los infantes, la organización educacional, las enfermedades y el cuidado de la salud, el bienestar social, el cuidado a los ancianos, la vivienda. Éstos son, a su vez, de importancia para la estructura y la composición de la producción y el comercio, los negocios y los servicios no industriales, tanto como para las costumbres de ahorro y la formación del capital.

Podemos citar finalmente el informe del ITM al respecto: “....consideramos que la exclusión del trabajo es una de las simplificaciones menos satisfactorias del modelo agregado, principalmente debido a las implicaciones sociales del desempleo. Aun cuando en la inclusión de una fuerza de trabajo explícita y la representación de las causas y consecuencias del desempleo es improbable cambiar el comportamiento básico del modelo "Mundo 3", estas condiciones lo harían más relevante para el estudio del bienestar social y de la estabilidad política.

\section{Los modelos васнU de la Oficina Internacional del Trabajo}

En 1972, la Oficina Internacional del Trabajo (orT), inició un proyecto de investigación de gran alcance, "Población y desempleo", dentro del marco del Programa Mundial de Empleo de la oIT. El objetivo final de este proyecto fue establecido en términos de "capacitar gobiernos, especialmente de países en desarrollo, para tomar en cuenta de manera más efectiva los temas de población en las políticas de empleo y viceversa".

Los medios para conseguir estos objetivos serían la construcción de un modelo que hiciera posible analizar las condiciones y las tendencias en la fuerza de trabajo y el empleo, así como los principales fenómenos y variables que la afectan. En otras palabras, es una cuestión de un modelo muy general en el cual, sin embargo, se podría dar por descontado que ciertos fenómenos tendrían papeles menos importantes que en los modelos del ITM. 
La primera etapa del trabajo desarrollado fue realizada por R. Blandy y R. Wery, en consulta con expertos del grupo de Sussex. El modelo está basado en las técnicas de análisis de sistemas. No se ha publicado aún un informe detallado, pero las principales características del proyecto ya han sido presentadas en algunos artículos preliminares.

El proyecto OIT, llamado luego La Diosa Bachue, consta de dos partes: a) una consiste en estudios que se ocupan de elementos importantes en la red de relaciones entre la población y el comportamiento del empleo. Paralelo a esto, se han hecho esfuerzos para construir modelos cuantitativos de simulación que reflejen esta red; $b$ ) el concepto inicial de un cistema económico-demográfico se formuló primero en un modelo llamado BACHUE-1. Éste es descrito como la primera y más importante experiencia de aprendizaje. El modelo está diseñado para representar una economía con una alta fecundidad en desarrollo. Diversos países se han comprometido a realizar aplicaciones específicas, el primero de los cuales es el BACHUE-Philippines.

Se espera que las experiencias futuras de estos modelos de países sirvan como una importante realimentación para el mejoramiento de las estructuras teóricas de BACHUE-1. De esta manera, los estudios sustantivos ayudarán al proceso de revisión sucesiva del modelo para entender los problemas de población-empleo en los países en desarrollo.

Dado que los modelos BACHUE emplean el método de análisis de sistemas, puede decirse en principio que tienen un gran número de ventajas y de desventajas o deficiencias, citadas antes en relación a los modelos del Iтм. Con respecto a los efectos de una fuerte agregación, debe mencionarse primero que éstos han sido reducidos de alguna manera, en parte restringiendo los objetivos y concentrándose únicamente en las áreas menos desarrolladas del mundo, en parte trabajando, en ciertas variables, a un grado de diferenciación mucho mayor.

Sin embargo, estas circunstancias conducen a otro tipo de dificultades. La cuantificación sólo puede ser hecha naturalmente con datos de países donde se disponga de datos confiables. Aquí, se ha preferido usar los datos de México, aunque se han usado también los datos de algunos otros países latinoamericanos. Esto produce evidentemente un sesgo cuando se trata de representar la totalidad del mundo menos desarrollado. Las diferencias en la estructura socioeconómica no están dadas como es debido.

Respecto a la metodología, aparecen dificultades en la medición y cuantificación de la migración y otros flujos dentro del subsistema económico. Sin embargo, un avance importante en los modelos BACHUE, es la forma en la cual los sectores rural y urbano de la población y la economía se encuentran separados.

El prototipo de los modelos BACHUE consiste de tres sectores:

1. El subsistema demográfico;

2. El subsistema educacional;

3. El subsistema económico.

Acerca del primero puede decirse que incluye la población por sexo, 
edad, lugar de residencia (rural/urbana) y actividad (empleo o desempleo). La mortalidad se expresa en forma muy simple por medio de la esperanza media de vida, a partir de la cual se obtienen tasas específicas de mortalidad con la ayuda del modelo de tablas de vida de Naciones Unidas (1956). A su vez, la esperanza de vida se supone que depende clel ingreso familiar medio, y aumenta desde una posición inicial en las regiones subdesarrolladas a un nivel correspondiente a las condiciones actuales de los países industriales.

En cuanto a la tasa de fecundidad, el modelo trabaja con dos componentes, el número deseado de nacimientos (más correctamente, de niños), e "incertidumbre". El número deseado o planeado se supone determinado por medio de los siguientes "modificadores": a) mortalidad infantil (positivo); b) nivel educacional medio de adultos (negativo); c) oportunidades para el trabajo femenino en los sectores modernos de trabajo (negativo) ; d) proporción de trabajadores familiares sin retribución en la fuerza de trabajo (positivo); e) nivel medio educativo de los niños (negativo); $\mathrm{y} f$ ) ingreso familiar medio (negativo).

Ésta es una aproximación bastante más diferenciada que aquella del modelo "Mundo 3" del ITM. Sin embargo, lleva consigo el riesgo de errores sistemáticos como resultado de la interacción entre estos factores de influencia. También, la cuantificación de una población-sociedad parece difícil, con riesgos y, en parte, imposible.

Por "incertidumbre" se entiende la efectividad de la práctica de planificación familiar. Se considera que ésta depende, entre otras cosas, del nivel educacional de las mujeres adultas. Se ha introducido una variable política como innovación que consiste en una tasa de difusión de la tecnología contraceptiva moderna, la cual se considera sujeta al control gubernamental. Un factor adicional es la diferencia entre lo urbano y lo rural, que es tomada en cuenta mediante un supuesto acerca de la adaptación en la migración.

Un método especial ha sido desarrollado para manipular la migración rural-urbana. La migración está basada, en primer lugar, en la propensión a migrar de los hombres en edades activas. La tendencia a migrar se considera afectada por el nivel educacional, el ingreso y las "variables relacionadas con el empleo".

La presentación de los modelos BACHUe contiene una discusión amplia, aunque no del todo clara, acerca de las definiciones de fuerza de trabajo en una sociedad en la que hay tanto un sector moderno como un sector tradicional. Lo significativo es que en los modelos anteriores sólo se tomó en cuenta conexiones causales unilaterales en los procesos económicodemográficos, mientras que el modelo BACHUE busca analizar "las interacciones económico-demográficas en un conjunto complejo de realidades cuya interconexión enfatiza el valor explicativo del modelo". Citaremos también que: "BACHUE-1" tiene tres rasgos distintivos: primero, es altamente endógeno: la fecundidad, la mortalidad, la migración rural-urbana, la propensión escolar, la productividad, la distribución del ingreso y los patrones de consumo - responden de manera endógena- al comporta- 
miento del sistema; segundo, involucra un alto nivel de desagregación. La población es dividida en grupos por sexo y edad y por status de actividad como en algunos modelos anteriores; su descomposición simultánea por lugar de residencia rural-urbana y la contemplación de niveles educacionales constituyen un rasgo nuevo. En forma similar, la economía está desagregada dentro de un esquema de gastos de cuentas nacionales con una matriz de insumo-producto que es usada para derivar el "valor agregado" sectorial y el empleo. Tal desagregación permite un análisis más riguroso del impacto de los factores de la oferta sobre la producción y el empleo y su retroalimentación sobre las variables demográficas; tercero, hace un intento explícito por presentar el dualismo económico (la coexistencia de sectores modernos y tradicionales) y de estimar el grado de distribución del ingreso personal... Simulaciones con el BACHUE-1 muestran que la reducción de la fecundidad debe ser uno de los factores fundamentales para reducir el dualismo económico.

El tratamiento de subsistema educacional está vinculado a un informe publicado por ocDE, "Un modelo de simulación del sistema educacional" (1970), en el cual se hace una división de acuerdo principalmente al número de años de escuela. Un sistema de circuitos de retroalimentación integran la educación dentro del sistema total. La combinación rural-urbano implica un claro mejoramiento si se compara con otros intentos hechos en modelos generales y sectoriales.

Es obvio que el modelo BACHUE incluye algunos factores y retroalimentaciones que no se encuentran en el modelo "Mundo 3", tanto en el sector población como en el económico. Además, aquí se incluye la educación.

Es muy pronto para revisar en esta presentación los detalles de BACHUE y de formarse una opinión de su valor para cubrir diversos propósitos. Sin embargo, podría verse que los mejoramientos sucesivos del modelo, mediante un diálogo planeado entre el modelo básico y los estudios sustanciales de países, harán posible obtener un instrumento útil de análisis y para la formulación de políticas en los tipos de países involucrados. Las dificultades de cuantificación son, no obstante, enormes.

Un siguiente paso sería combinar las experiencias y los modelos así obtenidos con una versión posterior del modelo "Mundo 3" del ITM. Esto no será tarea fácil, pero puede hacerse. Esto mejoraría, en efecto, el modelo BACHUE para satisfacer sus propios objetivos y ayudaría a producir un modelo general adecuado no sólo para países en desarrollo sino también para una variedad de sociedades homogóneas en relación con aspectos demográficos, sociales y otros.

Parece importante y urgente que las condiciones de salud (enfermedades agudas, invalidez e incapacidad para trabajar) sean incluidas explícitamente en el modelo. Se han realizado estudios especiales para establecer la metodología y el acopio de datos para esta ampliación. 


\section{Algunos otros modelos seleccionados}

Existe un buen número de modelos y proyectos de estudio que deben fundamentarse, relacionados en algunos aspectos con los temas de este informe, para que la extensión del método de los modelos pueda utilizarse para obtener mejores bases de decisión acerca de medidas políticas. En esta sección daremos cuenta breve de algunos de ellos.

Algunos de los proyectos son considerablemente más antiguos que los considerados en discusiones recientes acerca del futuro de la humanidad, mientras que otros han sido inspirados directamente por los modelos ITM - a menudo como un resultado de la crítica de estos últimos. Ciertos modelos son aproximaciones independientes que utilizan métodos específicos.

Aunque algunos modelos econométricos tienen un propósito general, por regla tienen algunas restricciones en lo que concierne a variables incluidas, tanto como a la forma y la medida de las interrelaciones entre varios fenómenos y factores. Estos modelos, por lo tanto, no responden a nuestra definición de modelos dinámicos, DES. Al mismo tiempo, sin embargo, es claro que los modelos relativamente simples pueden a veces hacer una contribución de valor directo en la interpretación del fenómeno, y por lo tanto, en teoría, pueden servir también como un método en la toma de decisiones.

Deberíamos mencionar aquí un grupo de modelos que no están construidos directamente como instrumentos para la toma de decisiones sino únicamente con el propósito limitado de proporcionar un panorama más adecuado de un fenómeno. En el sector población se encuentran así ciertos modelos de simulación que, colocados en un contexto más amplio, pueden servir como medios para el análisis de problemas sociales relacionados con la población, y aún ser incluidos como un subsector en un modelo general.

Puede enfatizarse aquí que los modelos mencionados en esta sección no forman sino una selección pequeña y no representativa, limitada por el poco tiempo disponible para publicar este informe.

En primer término, podemos mencionar un tipo de modelos de uso demográfico que han jugado un papel importante y aún lo hacen, en la planificación de países poco desarrollados donde no existe información precisa sobre las condiciones de la mortalidad. Un modelo especial de tablas de vida ha sido construido por las Naciones Unidas en la Oficina de Investigación de la Población, en Princeton, y por otras organizaciones. De esta serie de tablas de vida es posible, cuando se dispone de una o dos estimaciones del nivel de la mortalidad, seleccionar una tabla adecuada para el análisis y proyección de la población de un país específico. Pueden ser estimaciones adecuadas por ejemplo las de mortalidad infantil y las de algún intervalo de edades adultas. Esta técnica ha jugado un papel importante en la planificación nacional de tales áreas. Estas tablas de vida modelo han sido utilizadas también para otros propósitos, v. gr., como parte de modelos aproximados más generales.

Recientemente, se han hecho esfuerzos para construir series similares 
de tablas de fecundidad. De esta manera, aun en áreas con estadísticas poco desarrolladas, se pueden analizar y proyectar para un período posterior las condiciones demográficas. En la medida en que paulatinamente aparezcan o estén disponibles datos independientes, éstos métodos perderán su importancia. Por otra parte, tales tablas sirven para efectuar análisis de largo plazo relacionados con modelos más generales, y por lo tanto, constituyen un ingrediante importante en la construcción de modelos generales demográfico-económico-sociales.

En los últimos años, han sido desarrollados diversos modelos para simular la reproducción de algunos componentes. El esfuerzo pionero parece haber sido "La microsimulación de sistemas sociales", de G. Orcutt et. al., en 1961. Luego siguieron varios intentos, que parcialmente crearon modelos más detallados de fecundidad, y parcialmente para obtener modelos generales de todo el proceso reproductivo. Entre todos estos modelos, algunos pueden mencionarse aquí brevemente.

Un modelo determinístico con la denominación FERMOD desarrollado por R. Potter, M. Shops et al., ha sido utilizado para analizar entre otras cosas, los programas de fecundidad y planificación familiar. Más exhaustivo es el modelo estocástico popsim, desarrollado en el Triangle Research Institute. Una forma modificada de éste es el llamado REPSiM, de M. Shops et al. Ambas versiones pretenden hacer una descripción relativamente detallada de la reproducción de la población humana. Estos modelos han tenido un amplio campo de aplicación. Los modelos no han sido usados únicamente para analizar las condiciones corrientes de reproducción en distintas poblaciones sino también para comparar los diferentes métodos y proyectos de política con respecto al control de nacimientos.

Modelos de simulación de la fecundidad, en combinación con la nupcialidad y la mortalidad, han sido desarrollados después en el Instituto de Investigación Demográfica, de la Universidad de Gothenburg. Ciertas versiones de estos modelos tratan sobre las condiciones en las que no existe control de nacimientos, mientras que en otros modelos se toma en cuenta las condiciones de un país en donde existe control natal. Distintas funciones del control natal han sido estudiadas. Estos medios hacen posible simular la transición en circunstancias externas diferentes.

Un proyecto de investigación de la Universidad Católica de Lovaina en Le Bain (G. Wunsch y M. Loriaux) ha sido planeado para un modelo de simulación detallado y muy amplio. Éste pretende describir directamente el curso de la transición desde niveles elevados e incontrolados de mortalidad y fecundidad hasta niveles bajos y controlados, con lo que se intenta responder a la necesidad de los países en desarrollo de juzgar el curso de los eventos bajo diferentes consideraciones acerca de la efectividad del control de la mortalidad y fecundidad, respectivamente.

Como un ejemplo de modelo con propósitos limitados, podemos mencionar aquí un proyecto desarrollado en el "Centro de Estudios de la Familia y la Comunidad", en la Universidad de Chicago, cuyos autores son Donald J. Bogue, Scott Edmonds y Elizabeth J. Bogue. El proyecto es descrito en "Un modelo empírico para la evaluación demográfica del 
impacto de la contracepción y el status marital sobre la tasa de natalidad". (Manual 6, 1973). En él se incluyen aplicaciones estimadas para el establecimiento de metas y cuotas para programas de planeación familiar.

Aunque el modelo tiene alcances relativamente simples, es de gran flexibilidad. Lo que es particularmente importante es que permite manejar el proceso dinámico, en el tiempo, con todas las variables esenciales. Entre éstas se cuentan no sólo la fecundidad, mortalidad, migración y estado marital, sino también cuestiones acerca del control natal, tales como la efectividad de los métodos, el tiempo promedio de uso de las técnicas preventivas y la efectividad del uso real de los diferentes métodos. Si los últimos factores son tratados desde una perspectiva más general, es evidente la posibilidad de juzgar métodos alternativos para realizar programas de control de nacimientos en países con puntos de partida diferentes y objetivos variables. En suma, puede decirse que un modelo de esta clase puede ajustarse a los requerimientos de un sector dentro de un programa más general.

Como se apuntaba en la Introducción, varios proyectos de investigación han sido iniciados como consecuencia de la discusión y crítica que siguió a la publicación de "Los límites del crecimiento". Algunos fueron comenzados aún antes. Otros han desarrollado sus propios métodos para analizar los problemas del crecimiento.

La diversidad de estos proyectos hace imposible determinar, por el momento, lo que sería "el conocimiento integrado y coordinado" contenido en todos estos estudios. Éste es particularmente el caso, con unas cuantas excepciones, de informes de avances preliminares e incompletos que han sido publicados.

Desde el punto de vista de este informe, debe señalarse también que relativamente pocos de los proyectos intentan desarrollar modelos formales que puedan servir como "instrumentos de decisión" en el análisis y comparación de diferentes medidas políticas. Por otro lado, es evidente que muchos estudios en los que no se utilizan modelos, contienen observaciones y conclusiones de gran relevancia e importancia en la operación de modelos, y por ende, para la formulación última de políticas.

Nos limitaremos aquí a una breve mención de algunos estudios seleccionados. Los siguientes, se encuentran conectados directamente con el Club de Roma.

"Estrategia de supervivencia", por P. Pestel y M. O. Mesarivic. Este trabajo consiste en la construcción de un modelo jerárquico para decisiones políticas, en el cual se toman en consideración factores sociales, políticos y económicos.

El Instituto Battelle, en Génova, está llevando a cabo una modificación y desarrollo amplio de la técnica llamada DELPHI ("Dematel"). Los distintos elementos de los problemas del crecimiento, tal como son entendidos por los políticos y planificadores en las diferentes partes del mundo, se ponderan conjuntamente mediante una técnica especial y en la medida posible se cuantifican conexiones recíprocas.

Un grupo del llamado Club de Roma ha sido formado en Japón y tiene 
planeado realizar inicialmente, estudios amplios en diversas instituciones. Todos ellos trabajan en parte sobre los problemas del crecimiento en condiciones de bienestar, y en parte sobre el papel futuro de Japón en el mundo.

En América Latina se ha iniciado un gran proyecto para desarrollar un modelo mundial diferente, partiendo inicialmente, y en especial, de la situación y valoraciones de los países en desarrollo. El estudio es llamado "Primera alternativa de modelo mundial" y participan diversos países latinoamericanos bajo la dirección de Amílcar Herrera. El trabajo comienza con un análisis crítico del modelo del ITM, después de lo cual los autores diseñan su propio modelo. Se desarrollan hipótesis sobre el crecimiento y se hacen esfuerzos para estimar las posibilidades de desarrollo de los países subdesarrollados para un período de 30-35 años adelante. Los prospectos de igualdad económica entre países pobres y países ricos son juzgados, entre otras cosas, a la luz de las consecuencias sobre el medio ambiente.

Un proyecto de naturaleza especial es "El problema de duplicar la población", bajo la dirección de H. Linnemann con la asesoría de J. Tinbergen. En esencia, éste no es un modelo de construcción sistemática sino un análisis de los programas necesarios y de las decisiones que deben tomarse ahora, si vamos a manejar algunos de los problemas más difíciles de recursos bajo el supuesto de una población mundial duplicada.

\section{RESUMEN Y CONCLUSIONES}

Las críticas extensivas a que ciertos experimentos de modelos han estado expuestos por parte de científicos y técnicos seriamente comprometidos, así como por parte de individuos poco expertos, pero generalmente interesados y representativos de la clase media, no han sido del todo negativos. Se han sugerido diversos puntos de vista constructivos. Algunos de éstos han resultado en proyectos diseñados para mejorar, en ciertos puntos y subsectores, lo que ya ha sido publicado. En forma similar, se han lanzado ideas y métodos completamente nuevos para investigar e] desarrollo futuro.

El presente informe da cuenta del estudio de diversos modelos, descritos en algún grado, así como de un número limitado de revisiones críticas de tales modelos. Sin embargo, debe enfatizarse que la selección del material se vio seriamente restringida por el material que estaba realmente disponible durante un período corto.

A) Los modelos y los métodos elaborados hasta ahora, así como las tareas diferentes de expansión y mejoramiento de aquellos que se están zreando, no son todavía de una naturaleza y una capacidad que les permita funcionar como instrumentos en un análisis más profundo de los problemas demográficos-económicos-sociales. Por esta razón, no pueden vor el momento contribuir, en grado apreciable, a proporcionar mejores bases para decidir en la formulación y puesta en práctica de medidas de 
política en estas áreas. Aún menos pueden reemplazar partes de los procedimientos de investigación corrientes.

B) Sin embargo, aún en la situación presente, los modelos y los proyectos de modelos en proceso pueden - con uso crítico cuidadoso y tomando en cuenta las restricciones, generalizaciones y errores- ayudar a esclarecer la significación, a nivel macro, de las distintas tendencias en la sociedad actual, toda vez que éstas son inducidas a continuar en formas y direcciones fijas.

C) La investigación y el desarrollo posterior en este campo altamente técnico, requiere de recursos extensivos y de la cooperación de científicos de diferentes disciplinas. Lo que ha sido llevado a cabo hasta ahora se ha realizado parcialmente a través de actividades ocasionales organizadas en algunas pocas instituciones de investigación y a través de grupos de investigación organizados ad hoc. Sin embargo, se han hecho contribuciones valiosas por parte de institutos pequeños y por científicos individuales.

1. Para promover más el desarrollo de la investigación que se requiere, parece deseable hacer un arreglo para coordinar los proyectos ya existentes e iniciar la investigación sobre "gaps" en las fronteras de la investigación.

2. Además, es necesario garantizar la existencia continua de recursos financieros para todos los subproyectos. De otro modo, la ausencia de recursos financieros conduce a la distorsión de los esfuerzos de la investigación y el desempleo del grupo de investigación existente.

3. Debería notarse que estas tareas deben ser realizadas, en primer lugar, por Naciones Unidas y sus organismos especiales relacionados (ILO, WHO, UNESCO). Por supuesto diferentes formas de organización son posibles. Parece correcto que los proyectos de investigación ya existentes en las Universidades y otras organizaciones de investigación deberían coordinarse para utilizar al máximo a los grupos de investigación y los recursos financieros.

4. De manera independiente a cómo deba ser organizada y llevada a cabo la continuación del trabajo, la formulación, las aplicaciones y las cuantificaciones de los modelos deben hacerse, tanto como sea posible, por estratos (regiones, tipos de sociedad) homogéneos en sus aspectos esenciales.

5. En esencia, el desarrollo de un modelo más general que refleje los factores condicionantes y de cambio en la población y la sociedad, es sólo el primer paso.

$A$ priori, se puede pensar en el modelo como un sistema cerrado con distintas conexiones. Aunque el modelo sólo viene a ser de interés práctico, es posible expresar la influencia de variables exógenas sobre el problema central. Es por este medio que se puede esperar, a través de la construcción del modelo, acudir a este instrumento para analizar los efectos de medidas alternativas en los campos demográfico, económico, social y cultural.

Dado que los modelos están construidos, por naturaleza, con base en 
variables cuantitativas, deben encontrarse formas numéricas de las relaciones que expresan el efecto de las medidas tomadas; éstas tomarán la torma de cuadros, curvas o funciones matemáticas con conjuntos de parámetros. Un ejemplo de esto lo constituye la introducción de una variable de política en BACHUE, para el estudio de las actividades del gobierno en la planeación familiar.

El trabajo con modelos generales debe dirigirse especialmente hacia la producción de tales relaciones políticas. En la medida en que la cuantificación no pueda ser realizada, existe la posibilidad de trabajar con diferentes conjuntos de parámetros para formas alternativas de medidas $\mathrm{y}$ efectos alternativos del fenómeno. 